\title{
Development of a Sensitive Bead-Based Assay for Enhanced Monoclonal Antibody Detection
}

\author{
Manuel E. Ruidíaz ${ }^{1}$, Natalie Mendez ${ }^{2}$, Ana B. Sanchez ${ }^{3}$, Bradley T. Messmer ${ }^{3}$ and Andrew C. \\ $\mathrm{Kummel}^{4}$ \\ ${ }^{1}$ Department of Bioengineering, University of California, San Diego, La Jolla (UCSD), CA \\ 92093, U.S.A. \\ ${ }^{2}$ Division of Biological Sciences, UCSD, La Jolla, CA, 92093, U.S.A. \\ ${ }^{3}$ Moores Cancer Center, UCSD, La Jolla, CA 92093, U.S.A \\ ${ }^{4}$ Department of Chemistry and Biochemistry, UCSD, La Jolla, CA 92093, U.S.A
}

\begin{abstract}
Monoclonal antibodies are increasingly used in the treatment of cancer due to their enhanced targeting and immune system stimulation properties. Dosage guidelines typically do not account for personal cancer load or metabolism, thereby possibly affecting treatment outcome or causing unwanted side effects. The requirement for an assay that can quickly and precisely measure the concentration of the monoclonal antibody in a serum sample of a patient during therapy is unmet. A bead-based assay with peptide antigen mimetics has been developed to rapidly determine the concentration of antibody drug present in serum specimens with high sensitivity. Alemtuzumab (anti-CD52) and rituximab (anti-CD20) antigen mimetic peptides, as discovered by phage display, were synthesized on 10 um TentaGel resin beads using conventional solid phase peptide synthesis techniques. The beads were modified to allow for multiplexing and microfluidic handling via fluorescent labeling and magnetic functionalization. The antigen-displaying fluoromagnetic particles were incubated with spiked serum samples which allowed free antibody to be captured. Primary antibody detection was performed on alemtuzumab while rituximab detection was used to compensate for non-specific serum binding to the beads. After washing, the beads were incubated with a fluorescently tagged secondary label for detection by flow cytometry. (Results) A fast, low cost, specific assay has been developed with several key techniques which allows detection at low concentration $(0.1 \mathrm{ug} / \mathrm{ml})$ of spiked samples. Primary to achieving this detection limit was the implementation of a compensation scheme where two antigen mimetic peptides behave linearly $\left(\mathrm{R}^{2}=0.996\right)$ which enables the calculation of the zero response of the antigen mimetic peptide of interest (alemtuzumab antigen mimetic) while measuring the zero response of the compensatory antigen mimetic peptide (rituximab antigen mimetic) during primary assay measurement. This reduces fluorescence response variation due to variations present due to sample preparation, storage and different patients because of the equivalent interactions these effects have on the compensatory beads. The developed assay is therefore robust against serum variation and enables a lower limit of detection.
\end{abstract}

\section{INTRODUCTION}

Monoclonal antibodies are a growing class of therapeutics used in the treatment of various cancers and auto-immune disorders (1-6). Traditional monoclonal dosage regiments 
typically do not take into account the specific tumor load or physiology of the patient (7-9) therefore it is hypothesized that an improper dosage may occur. Patients with a high intrinsic tumor load my have a low dosage relative to the amount of cancer that is present in that patient. Conversely, a patient with a lower tumor load, may be overdosed. Typical techniques used for detection of drugs from human serum samples are almost always difficult to perform and often have a high cost that makes it prohibitive to perform on every patient (10-13). Therefore, the need for a quick, low cost, assay to reliably determine the levels of monoclonal antibody drugs is unmet $(10,11)$. Herein, we describe a system to quickly determine the level of monoclonal antibody levels in human serum samples. Several techniques have been integrated into the assay to account for variable non-specific binding of different patient serums. Additionally the assay platform has been engineered for compatibility with automated sample handling systems.

\section{EXPERIMENT}

\section{$\underline{\text { Bead Preparation }}$}

Alemtuzumab and rituximab antigen mimetic peptides sequences were generated by phage-displayed peptide library screening and synthesized on $10 \mu \mathrm{m}$ Tentagel beads as described previously (14). Automated handling and multiplexing capability were enabled on the bead platform by the trapping of nanoparticles within the polystyrene matrix of the bead. For automated handling capability, magnetic nanoparticles can be incorporated to enable handling of beads by magnetic field thereby eliminating the requirement for centrifugation during the assay and enabling control by automated means. For multiplexing of multiple beads types, fluorescent nanoparticles with different emission wavelengths can be similarly incorporated within the beads allowing them to be identified by dependent on which emission wavelength is detected.

A two phase process is used to incorporate the dye and/or the magnetic material which involves the mixture of two separate solutions, a swelling solution and an incorporation solution. Swelling Solution: $5 \mathrm{mg}$ of peptide-beads were dissolved in 500ul N-N-dimethylformamide (DMF) in a microcentrifuge tube followed by sonication in a Branson 2510 bath sonicator (Branson Ultrasonic Corp., Danbury, CT) at room temperature until beads were visibly dissolved and dispersed. Incorporation Solution: A 500ul aliquot of DMF was placed into a microcentrifuge tube. For magnetization, 200ul of $6.5 \pm 3.0 \mathrm{~nm}$ iron oxide $(\mathrm{FeO})$ magnetic nanoparticles (MagNP) dissolved in heptane (Fluka Chemika, Buchs, Switzerland) was added creating a DMF/heptane-MagNP dual phase system. A small hand-held neodymium magnet was positioned below the microcentrifuge tube causing the MagNP in the heptane phase to migrate to the DMF phase. The heptane phase was remove and discarded. The resultant DMF-MagNP is placed into a bath sonicator until MagNP were dispersed into the DMF.

For fluorescence multiplexing, $3 \mathrm{ul}$ of $40 \mathrm{~nm}$ red (alemtuzumab-binding beads) or dark red (rituximab-binding beads) fluorescent FluoSpheres carboxylate-modified microspheres (Invitrogen Corp., Carlsbad, CA) was added to the DMF solution. This was followed by sonication at room temperature until the beads were dispersed. The 500ul incorporation solution was transferred to the 500ul swelling solution tube. The combined solution was sonicated for 10 minutes to assure dispersion and incorporation of the nanoparticles within the polystyrene bead matrix. The beads in solution were centrifuged for 2 minutes at 14,000 rpm (Microfuge 18 Centrifuge, Beckman Coulter, Mount Holly, NJ). The resultant supernatant was discarded and 

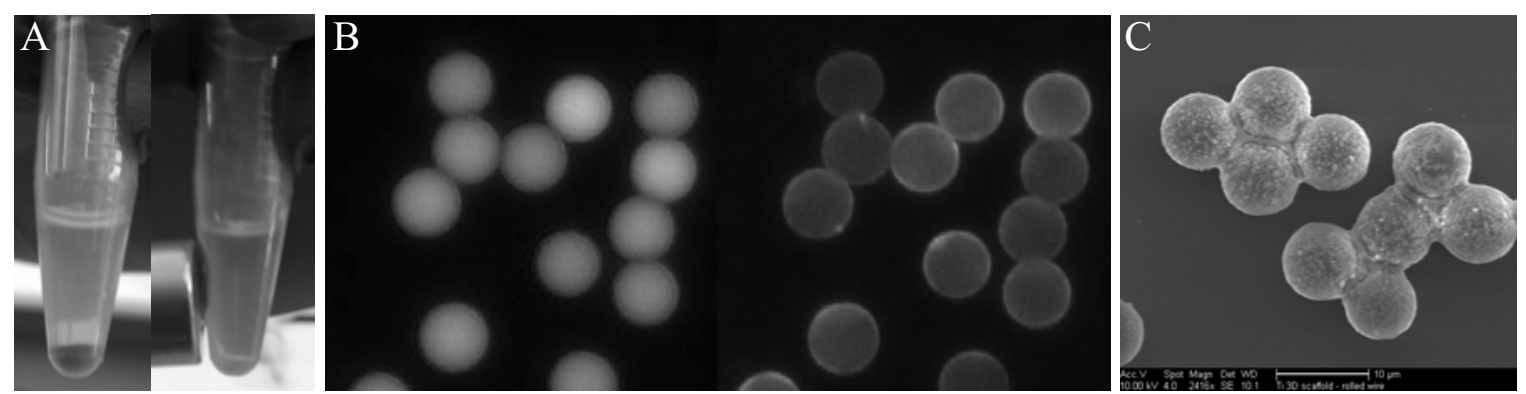

Figure 1: (Left) Modified fluoromagnetic beads reacting to magnetic field. (Middle) Two color fluoromagnetic beads after incubation in $10 \mathrm{mg} / \mathrm{ml}$ alemtuzumab and secondary showing red beads with green response channels respectively indicative of attached alemtuzumab. (Right) Scanning electron micrographs of fluoromagnetic particles with salt crystallization artifact.

the pellet was resuspended in phosphate buffered saline (PBS) (Invitrogen Corp., Carlsbad, CA). Sonication and centrifugation followed by resuspension with PBS was repeated two additional times and finally resuspended with PBS with $0.05 \%$ sodium azide (Teknova, Hollister, CA) and stored at $4^{\circ} \mathrm{C}$. Bead characterization is detailed in Figure 1.

\section{Monoclonal Antibody Measurement Protocol}

BD Falcon 96-well polystyrene V-bottom plate wells (Becton, Dickinson and Company, Franklin Lakes, NJ) were coated with a 3\% solution of $\mathrm{IgG} /$ protease free Bovine Serum Albumin (BSA) (Jackson ImmunoResearch Laboratories, Inc., West Grove, PA) in PBS with $0.05 \%$ sodium azide to reduce non-specific binding to the well surfaces. The remaining BSA in the wells was aspirated and discarded. To each well, a primary antibody incubation solution was added which consisted of $100 \mathrm{ul}$ of $3 \%$ BSA solution, 10ul of PBS with a specific concentration of monoclonal alemtuzumab antibody as obtained from the UCSD cancer center pharmacy (Genzyme, Cambridge, MA), 10ul of human serum, purchased from Innovative Research (Novi, MI) and Valley Biomedical Inc (Winchester, VA), with an "unknown" concentration of monoclonal antibody and $3 \mathrm{ul}$ of a $1: 1 \mathrm{mixture}$ of $5 \mathrm{mg} / \mathrm{ml}$ alemtuzumab-binding beads and $5 \mathrm{mg} / \mathrm{ml}$ rituximab-binding beads in PBS. Samples were incubated for $1 \mathrm{hr}$ on a rocking platform shaker at room temperature. The samples were washed 3 times by magnetic capturing of the beads on the well bottom and replacing the supernatant with 100ul of the 3\% BSA-azide solution. The samples were incubated with $0.5 \mathrm{ul}$ of $2 \mathrm{mg} / \mathrm{ml}$ Alexa Fluor 488 conjugated Protein $\mathrm{G}$ (Invitrogen, Carlsbad, CA) in PBS with 99.5ul of 3\% BSA-azide solution for $1 \mathrm{hr}$ on shaker at room temperature. The samples were washed 3 times with 3\% BSA-azide solution and finally resuspended in 100ul of 3\% BSA-azide. Beads were then measured on a BD FACSCalibur (Becton, Dickinson and Company, Franklin Lakes, NJ) and the data was analyzed and gated using FlowJo (Treestar, Ashland, OR) software. Limit of detection was performed using the protocol described previously (14).

\section{DISCUSSION}

\section{Compensation for Non-specific Binding}

Fluorescence analysis of 10 different serums samples on 2 time points separated by 15 weeks demonstrated an 8 fold variation in the alemtuzumab fluorescence at zero concentration, 
indicating a time dependent non-specific background response in addition to the serum dependent background response changes (Figure 2). Therefore a technique that can independently determine the level of non-specific background response for the alemtuzumab was developed by including an additional bead with a peptide for an antibody (rituximab) known not to be present in the sample of interest. As shown in Fig 1, the non-specific binding on the peptides for the two antibodies is highly correlated so it is a reasonable basis for developing a method for non-specific background response determination in a large range of samples. The increase in background fluorescence of the alemtuzumab-binding peptide was determined to be linearly related $\left(\mathrm{R}^{2}=0.996\right)$ to the increase in the background fluorescence of the rituximabbinding peptide (Figure 1). This enables a method of zero determination of alemtuzumab samples without having to obtain samples that do not have alemtuzumab.

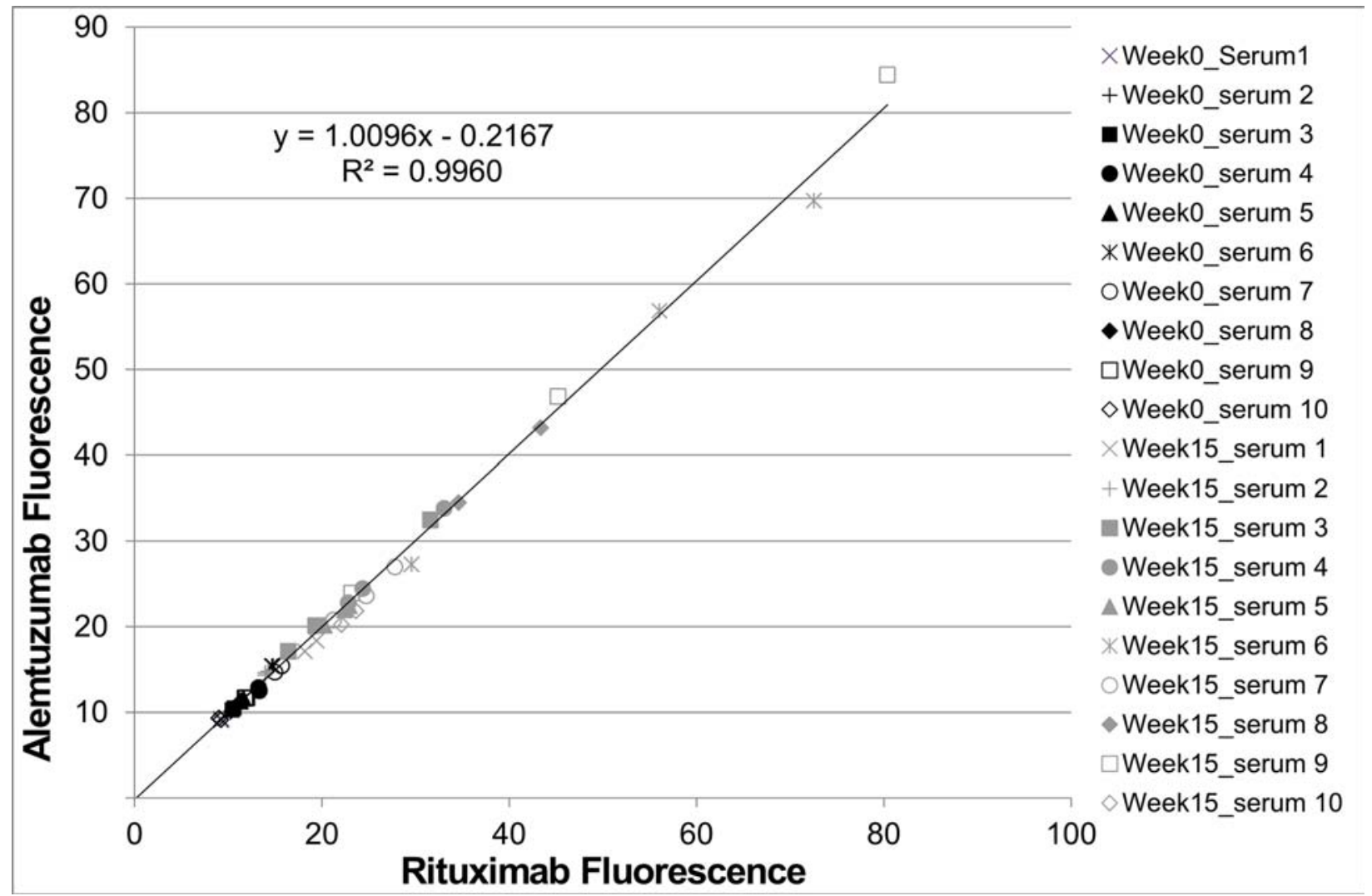

Figure 2: Non-specific background fluorescence values of 10 serum samples showing time dependent and serum dependent response. Changes in alemtuzumab-binding bead fluorescence are correspondingly exhibited in the rituximab-binding bead fluorescence. Black points are at week 0 , grey points are at week 15 .

\section{Monoclonal Antibody Measurement Assay}

"Unknown" serum samples were created as a surrogate for clinically relevant serum samples that would be obtained during ongoing monoclonal antibody therapy. To determine the fluorescence response for a given serum with an unknown alemtuzumab concentration, three different spike concentrations of alemtuzumab was added to three separate samples in addition to the unspiked sample. These four samples were performed in 4 replicates. Alemtuzumab-binding 
bead and rituximab-binding bead fluorescence response was measured by flow cytometry. For each sample, the fluorescence intensity of the rituximab-binding beads was used to subtract the non-specific binding of the corresponding alemtuzumab-binding beads. The resultant compensated curve was used to determine the original "unknown" concentration of alemtuzumab in the sample.

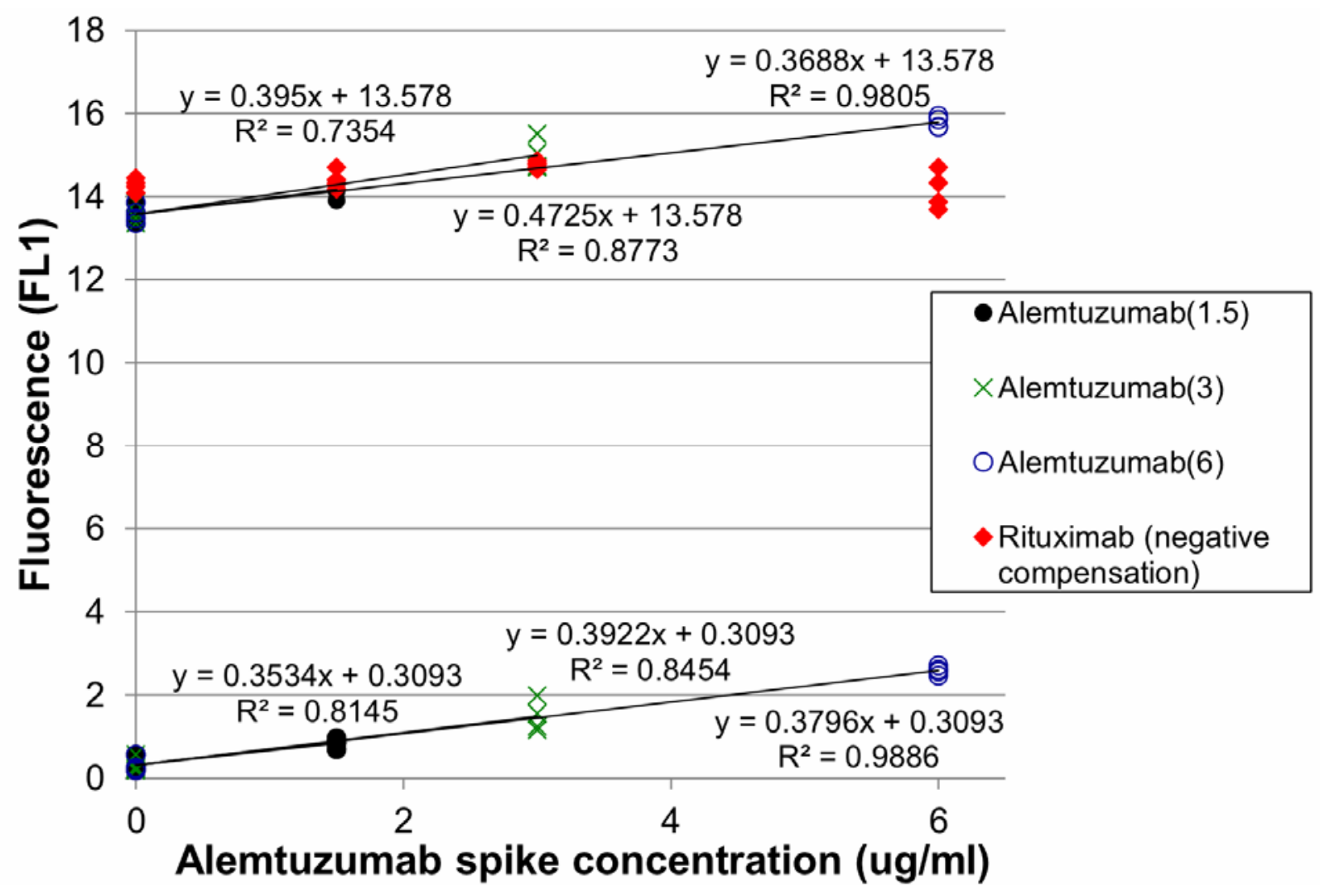

Figure 3: The above linear relationship is the uncompensated alemtuzumab-binding bead fluorescence response. Using the negative background response compensation rituximab-binding beads, a compensated alemtuzumab-binding bead fluorescence response is calculated below. The "unknown" alemtuzumab concentration was $0.5 \mathrm{ug} / \mathrm{ml}$ and the measured alemtuzumab concentration is $0.83 \mathrm{ug} / \mathrm{ml} \pm 0.044 \mathrm{ug} / \mathrm{ml}$ (Standard Deviation).

A determination of the limit of detection was performed on a serum sample with 7 spiked alemtuzumab concentrations $0,0.1,0.5,1,2,4,8 \mathrm{ug} / \mathrm{ml}$ on 3 different days in quadruplicate and was determined to be $0.5 \mathrm{ug} / \mathrm{ml}$, as determined by the lower $95^{\text {th }}$ percentile above zero. An ELISA based assay limit of detection based on a 6 samples performed over 4 different days was determined to be $1 \mathrm{ug} / \mathrm{ml}(14)$.

Three key advantages of this assay platform are exploited to successfully detect antibody at low concentration: (1) The ability to determine the level of background non-specific binding of the antibody of interest by determining the background level of another peptide known to have zero specific binding activity, (2) the presence of the polyethylene glycol (PEG) subgroup inherent in the Tentagel bead provides the platform with the low background necessary to 
provide detection at physiologically relevant concentrations, and (3) the porous nature of the Tentagel bead structure allows for multiple binding sites to be available to the antibody thereby allowing a cooperative binding effect which further increases the sensitivity of the assay.

\section{CONCLUSIONS}

A promising platform for low concentration monoclonal antibody detection has been presented which is able to compensate for donor serum, experimental, time based non-specific background variation. The versatility of the platform allows for the incorporation of magnetic and fluorescent nanoparticles which impart multiplexing and automated handling capability. As currently implemented, the protocol calls for a 1:10 dilution of the serum to be analyzed. Therefore the beads are physically reporting an order of magnitude reduced concentration of antibody. With further enhancement it is believed that the sensitivity of the assay may be improved at least 5 fold.

\section{REFERENCES}

1. J. M. Reichert, V. E. Valge-Archer, Nat Rev Drug Discov 6, 349 (2007).

2. M. v. Mehren, G. P. Adams, L. M. Weiner, Annual Review of Medicine 54, 343 (2003).

3. J. S. Ross et al., American Journal of Clinical Pathology 122, 598 (2004).

4. $\quad$ M. Harris, The Lancet Oncology 5, 292 (2004).

5. K. Arzoo, S. Sadeghi, H. A. Liebman, Annals of the Rheumatic Diseases 61, 922 (2002).

6. S. Perrotta et al., British Journal of Haematology 116, 465 (2002).

7. $\quad$ S. Demko, J. Summers, P. Keegan, R. Pazdur, Oncologist 13, 167 (2008).

8. R. M. Giusti, K. A. Shastri, M. H. Cohen, P. Keegan, R. Pazdur, Oncologist 12, 577 (2007).

9. M. H. Cohen, Y. L. Shen, P. Keegan, R. Pazdur, Oncologist 14, 1131 (2009).

10. T. J. Kipps, B. T. Messmer, A. B. Sanchez, A. C. Kummel, M. Ruidiaz, C. The Regents of the University of, Ed., vol. PCT/US2009/038674.

11. C. W. N. Damen, E. J. B. Derissen, J. H. M. Schellens, H. Rosing, J. H. Beijnen, Journal of Pharmaceutical and Biomedical Analysis 50, 861 (2009).

12. H. Blasco et al., Journal of Immunological Methods 325, 127 (2007).

13. G. Hale et al., Blood 104, 948 (2004).

14. A. B. Sanchez et al., Cancer Chemotherapy and Pharmacology 66, 919 (2010). 